\title{
Ellipsométrie spectroscopique
}

L'ellipsométrie est une technique optique précise, consistant à mesurer la modification de la polarisation d'un faisceau à la réflexion sur une surface ou un échantillon. Cette technique d'analyse et de caractérisation, non destructive, est précise et très sensible. La mesure ellipsométrique comporte trois étapes indispensables: l'étalonnage de l'instrument, la mesure proprement dite et l'analyse des données.

L'ellipsométrie spectroscopique effectue des mesures sur tout un spectre et concerne des structures complexes: multicouches, rugosité (interface, homogénéité...). Les domaines d'application de l'ellipsométrie sont de plus en plus larges. Elle s'annonce déjà comme un outil de prédilection des nanotechnologies. Par ailleurs de nombreuses applications sont envisagées telles que la photoélasticimétrie en mécanique, I'optique cristalline, la saccharimétrie en chimie, la mesure de l'effet Faraday en magnétisme et la mesure des effets Kerr en électricité. Nous présentons dans cet article la technique spectroscopique et ses diverses applications.

\section{N. GHELLAI, A. BENMANSOUR, \\ N.-E. CHABANE SARI}

Laboratoire de Matériaux et des Énergies Renouvelables

Université Abou Bekr BELKAIID

BP : 119 TLEMCEN - 13000 Algérie

Tel. /Fax : 043.21.58.89 \& 90

na_ghellai@yahoo.fr

L es méthodes optiques permettent de caractériser un très grand nombre de paramètres: concentration des porteurs, présence d'impuretés, propreté d'une surface. Elles présentent l'avantage sur les méthodes électriques, d'être non destructives et de ne pas exiger la réalisation, toujours délicate, de contacts ohmiques. L'ellipsométrie est une méthode de caractérisation optique, basée sur l'analyse de l'état de polarisation (souvent elliptique) d'une lumière initialement polarisée après sa réflexion sur une surface [1]. Son principe a été découvert il y a plus d'un siècle [2] et a trouvé un essor récent grâce à l'utilisation des micro-ordinateurs et de la comman de électronique des moteurs, ce qui a permis I'automatisation et l'optimisation des mesures [3].

Les principaux atouts de cette technique sont son caractère non destructif qui autorise des mesures in situ $[1,3]$; sa sensibilité qui permet de mesurer des épaisseurs jusqu'à une fraction de couche moléculaires
$[1,3]$, ainsi que la possibilité de suivre la croissance d'une couche en temps réel [3]. Il faut distinguer l'ellipsométrie à une seule longueur d'onde, utilisée pour un système monocouche, laquelle ne permet l'identification que de deux paramètres (épaisseur et indice de réfraction), de l'ellipsométrie spectroscopique qui effectue des mesures sur tout un spectre de longueurs d'onde, permettant l'étude de structures complexes : multicouches, rugosité d'interface, l'homogénéité, etc. $[3,4]$. Son utilisation récente dans plusieurs domaines s'explique par :

Le besoin grandissant de techniques non destructives permettant de travailler in situ et de grande sensibilité.

La nécessité d'observer dans une large gamme de mesure. En effet, on mesure l'épaisseur d'une fraction de couche monoatomique jusqu'à quelques micromètres.

Un perfectionnement général de l'appareillage, aussi bien les composants opti ques, la détection ainsi que le traitement du signal.

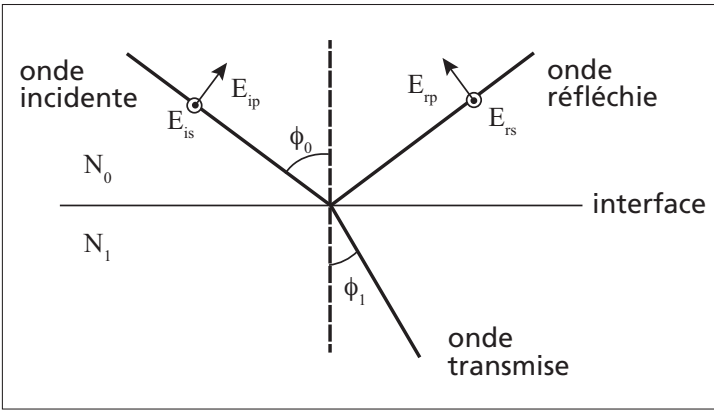

L'introduction de l'informatique, ce qui a permis la conception d'appareils automatisés, donc très rapides et très performants, et des modélisations théoriques très complexes.

\section{Principe de base de l'ellipsométrie}

\section{Principe de la mesure}

Considérons une onde électromagnétique plane polarisée arrivant sur l'interface, supposée plane, entre deux milieux (0) et (1) (cf. fig. 1). Une partie de l'onde est transmise ou "réfractée», et une autre partie est réfléchie. Le plan de la figure 1 est appelé plan d'incidence. Le champ électrique $E_{i}$ de l'onde incidente peut être écrit comme la somme de deux composantes orthogonales: la première, appelée polarisation $\mathbf{p}$, d'amplitude complexe $E_{i p}$, est dans le plan d'incidence et la seconde, appelée polarisation $\mathbf{s}$, d'amplitude complexe $E_{i s,}$ est orthogonale au plan d'incidence [1]. On peut décomposer de la même façon le champ électrique $E_{r}$ et le champ transmis $E_{t}$. Nous n'étudierons ici que l'onde réfléchie, mais il est possible de définir également des coefficients de transmission [1].

Figure 1. Réflexion sur une interface de Fresnel. 
Les coefficients de réflexion $r_{p}$ et $r_{s}$ sont des grandeurs complexes et correspondent respectivement aux polarisations $p$ et $s$ :

$r_{P}=\frac{E_{r p}}{E_{i p}}=\left|r_{p}\right| e^{j \delta p}$ et

$r_{s}=\frac{E_{r s}}{E_{i s}}=\left|r_{s}\right| e^{j \delta s}$

Où $\left|r_{p}\right|$ et $\left|r_{s}\right|$ sont les modules $\delta_{s}$ et $\delta_{p}$ et les phases de $r_{p}$ et $r_{s}$ (et $j$ l'imaginaire pur unité). On définit également la réflectivité pour la polarisation $p, R_{p}$ et la réflectivité pour la polarisation $s_{,} R_{s}$ par les relations :

$R_{p}=\left|r_{p}\right|^{2}$ et $R_{s}=\left|r_{s}\right|^{2}$

Par définition, l'indice de réfraction complexe $\mathrm{N}$ d'un milieu est :

$\mathrm{N}=\mathrm{n}+\mathrm{jk}$

Où $n$ est l'indice de réfraction réel $d u$ milieu considéré, et $k$ son coefficient d'extinction [1].
Si l'interface entre (0) et (1) est une interface de Fresnel, c'est-à-dire parfaitement plane et infiniment mince et si l'on note $\phi_{0}$ l'angle (complexe) d'incidence, $\phi_{1}$ l'angle (complexe) de réfraction, et $\mathrm{N}_{0}$ et $\mathrm{N}_{1}$ les indices de réfraction complexes des milieux (0) et (1), alors la loi de Descartes de la réfraction s'écrit $[1,2]$ :

$\mathrm{N}_{0} \sin \phi_{0}=\mathrm{N}_{1} \sin \phi_{0}$

De plus, toujours dans I'hypothèse d'une interface de Fresnel, les coefficients de réflexion peuvent être déduits de la continuité des composantes tangentielles des champs électrique $\mathbf{E}$ et magnétique $\mathbf{H}$ à l'interface [1]

$$
\begin{aligned}
& r_{p}=\frac{N_{1} \cos \phi_{0}-N_{0} \cos \phi_{1}}{N_{1} \cos \phi_{0}+N_{0} \cos \phi_{1}} \text { et } \\
& r_{s}=\frac{N_{0} \cos \phi_{0}-N_{1} \cos \phi_{1}}{N_{1} \cos \phi_{0}+N_{1} \cos \phi_{1}}
\end{aligned}
$$

Après la réflexion de L'onde électromagnétique sur la surface, elle se transforme en une onde elliptique (fig.1). L'ellip- somètre consiste à analyser cette ellipse par l'intermédiaire du rapport $\rho$ (la quantité mesurée) des coefficients de réflexion parallèle (p) et perpendiculaire (s).

$\rho=\frac{r_{p}}{r_{s}}=\left|\frac{r_{p}}{r_{s}}\right| e^{i\left(\delta_{p}-\delta_{s}\right)}$

On introduit souvent les angles ellipsométriques $\psi$ et $\Delta$, définis par [1] :

$\rho=\tan \psi e^{i \Delta}$

Les équations (I.1) permettent d'écrire :

$\tan \psi=\frac{\left|r_{p}\right|}{\left|r_{s}\right|}$ et $\Delta=\delta_{p}-\delta_{s}$

$\psi$ et $\Delta$ sont appelés les angles ellipsométriques. Ce sont les valeurs mesurées par notre instrument. La mesure des deux paramètres ellipsométriques $\psi$ et $\Delta$ ( $\tan \psi$ et $\cos \Delta$ ) permet d'obtenir les propriétés optiques du système réfléchissant. L'ellipticité $\varepsilon$ est donnée par le rapport du grand axe et du petit axe.

Tan $\psi$ est lié à ce rapport. L'angle de rotation $\Theta$ entre le grand axe et l'axe de

\section{(ind LFESER Fis 2111 Laser2000 distributeur exclusif The future of Photonics des lasers à fibre picosecondes accordables de Genia Photonics}

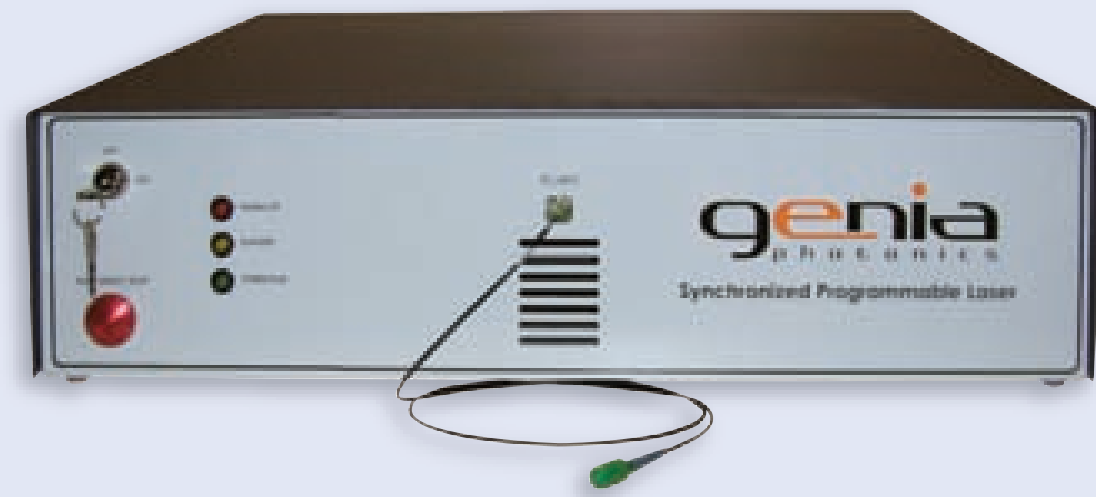

Le laser programmable est une source picoseconde compacte entièrement contrôlable par une interface PC. Tous les paramètres tels que la longueur d'onde, la vitesse de balayage, la durée d'impulsion ps, et la puis- sance peuvent être ajustés en quelques microsecondes pendant le fonctionnement du laser. II couvre les gammes spectrales $1030-1130 \mathrm{~nm}$, $1250-1360 \mathrm{~nm}$ et $1525-1605 \mathrm{~nm}$ et délivre jusqu'à $100 \mathrm{~mW}$ de puissance moyenne.
Le laser programmable et synchronisé est un laser programmable impulsionnel picoseconde couplé à un amplificateur de type MOPA et disposant d'une fibre de sortie unique dans un design compact et robuste. Cet outil permet de contrôler simplement, grâce à l'interface informatique, les délais entre deux impulsions consécutives.

Applications : spectroscopie CARS, SRS,

\begin{tabular}{|c|}
\hline Laser2000 \\
\hline \begin{tabular}{|l} 
Rémy CARRASSET \\
Tél. : +33130 802341 \\
carrasset@laser2000.fr
\end{tabular} \\
\hline
\end{tabular}
optique non-linéaire, $\mathrm{OCT}_{1}$.. 


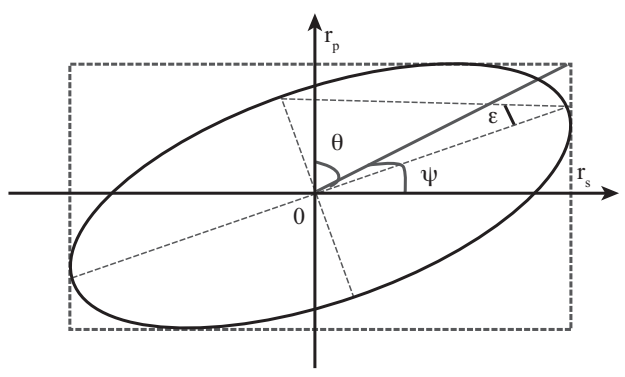

Figure 2. Polarisation elliptique de la lumière réfléchie.

polarisation $P$; $\Delta$ est lié à cet angle de rotation.

La mesure des deux paramètres ellipsométriques $\psi$ et $\Delta(\tan \psi$ et $\cos \Delta)$ permet d'obtenir les propriétés optiques du système réfléchissant [4] :

$N_{1}=N_{0} \tan \Phi_{0} \sqrt{1+\left(\frac{1-\rho}{1+\rho}\right)^{2} \tan \Phi_{0}{ }^{2}}$

$\Phi_{0}$ : Angle d'incidence, $\Phi_{1}$ : Angle de réfraction.

$N_{0}$ le coefficient du milieu extérieur et $N_{1}$ le coefficient de réfraction de l'échantillon.

\section{Les différents ellipsomètres}

Tout ellipsomètre se compose d'une source lumineuse $(L)$, d'un polariseur $(P)$, l'échantillon à étudier $€$, un analyseur pour déterminer l'état de polarisation après réflexion (ou transmission) de l'échantillon (A) et finalement d'un détecteur (D) pour déterminer l'intensité.

\section{Méthode de zéro: ellipsométrie à annulation}

Le schéma de base d'un ellipsomètre à extinction est donné par la figure 3.

La polarisation, linéaire après le polariseur, est transformée en polarisation elliptique par le compensateur. On oriente ce dernier de manière à obtenir une polarisation linéaire après réflexion sur l'échantillon. L'analyseur est par la suite orienté de manière à être croisé avec la polarisation linéaire obtenue jusqu'à l'extinction du faisceau.

\section{Méthode de modulation par élément tournant}

Le faisceau peut être modulé en polarisation par la rotation du polariseur, de

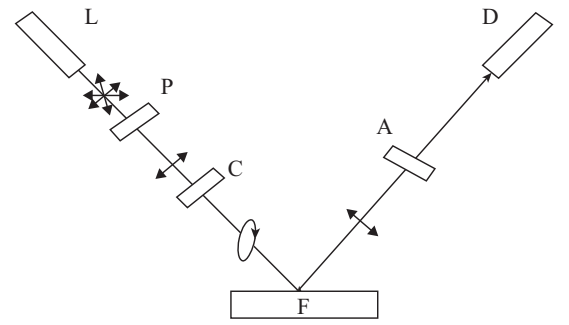

Figure 3. Ellipsomètre à annulation.

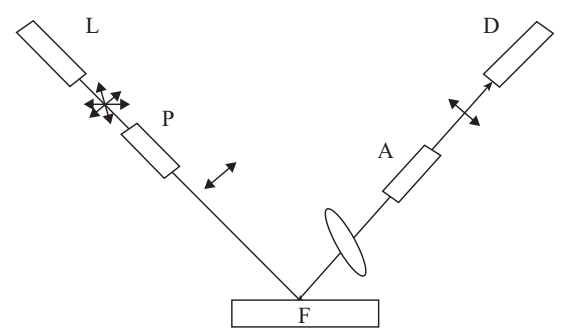

Figure 4. Ellipsomètre à modulation.

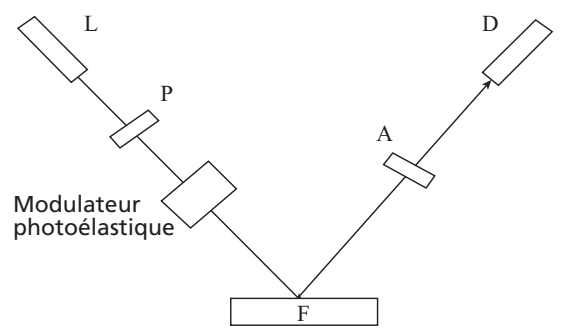

Figure 5. Ellipsomètre à modulation de phase.

I'analyseur ou du compensateur. La détection se fait à la fréquence de modulation par analyse numérique du signal détecté.

a) Un ellipsomètre à polariseur tournant nécessite une source dont la polarisation est entièrement définie. L'échantillon et l'analyseur étant fixes, on place un spectromètre entre l'analyseur et le détecteur pour disperser et filtrer la lumière parasite présente au niveau de l'échantillon. Tech nique relativement facile, nécessite l'alignement des composantes optiques pour atteindre un bon niveau de précision.

b) Un ellipsomètre à analyseur tournant implique au contraire l'emploi d'un dé tecteur insensible à l'état de polarisation. Cela impose dans ce cas de placer le spectromètre entre la source et le polariseur. Le détecteur sera sensible à la lumière parasite.

c) Un ellipsomètre à compensateur tournant permet de s'affranchir de toutes les contraintes de polarisation au niveau de la source et du détecteur mais nécessite un étalonnage spectral du compensateur, ce qui est source d'erreurs systématique sur la mesure.

\section{Méthode à modulation de phase}

Le montage optique est le même que le précédent (source, polariseur, analyseur, détecteur) et on ajoute un modulateur après le polariseur (voir fig. 5).

Dans cette configuration aucune caractéristique n'est requise au niveau de la polarisation pour la source et le détecteur.

La méthode à modulation de phase ne nécessite pas un alignement très précis car on n'a pas d'élément en rotation, mais nécessite une électronique très performante, capable d'assurer la saisie du signal et son traitement à une fréquence compatible à $50 \mathrm{KHz}$.

L'avantage de cette technique est que toutes les composantes optiques sont fixes durant les mesures, ce qui nous affranchit des problèmes de fluctuations mécaniques. De plus, la fréquence de modulation étant typiquement de $50 \mathrm{kHz}$, elle permet d'éviter les perturbations par les bruits habituels d'un laboratoire (pompes, réseau électrique...).

\section{Les paramètres mesures et interprétations}

L'ellipsométrie ne donne que des informations indirectes. Une étape d'analyse et de régression est indissociable de la mesure. Dans le cas d'un système multicouches, il est impossible de remonter directement à une couche donnée. II faut faire l'hypothèse d'une structure, en calculer la réponse, puis la comparer aux mesures.

L'exploitation des résultats consiste à analyser les spectres obtenus et à les confronter au modèle de l'échantillon mesuré. Grâce à l'informatique (traitement du signal ; bases des données d'indices de matériaux et de modèles; algorithmes de régression ; calcul matriciel...), on ajuste les paramètres théoriques et on remonte aux valeurs effectives de l'épaisseur et $d^{\prime}$ indices optiques. 


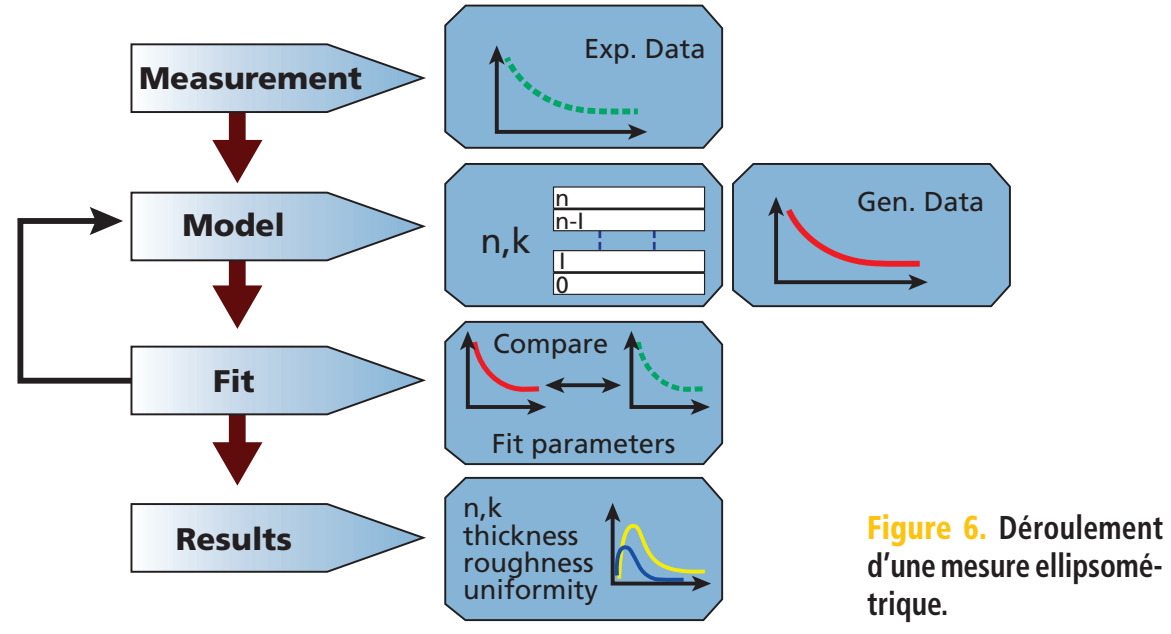

Les avantages de l'ellipsométrie spectroscopique sont :

- Le caractère non destructif (il n'y a ni perturbation ni interaction avec le matériau mesuré).

- La large gamme des mesures : I'épaisseur de la couche étudiée s'étend sur un large domaine allant du nanomètre jusqu'à quelques microns.

- La possibilité de contrôle « in situ » $[7,8]$.

- L'accès selon les configurations optiques à de nombreux paramètres (épaisseur, indices, porosités, liaisons chimiques...). Les inconvénients de cette technique de métrologie optique sont :

- La limite en résolution latérale est expliquée par la taille du faisceau lumineux qui est de l'ordre de quelques millimètres. La taille du faisceau peut être réduite à 50 um pour faire des balayages en XY.

- La nécessité d'une bonne connaissance du modèle physique des matériaux étudiés.

- L’homogénéité en épaisseur de la couche mesurée.

- L'état de la surface et/ou de l'interface d'un échantillon joue un rôle important : les mesures doivent être faites sur des zones planes.

- L'ellipsométrie est une méthode indirecte, elle ne donne pas accès directement à des paramètres physiques de l'échantillon.

\section{Domaines d'application}

L'ellipsométrie spectroscopique trouve ses applications dans plusieurs domaines: - L'optique : caractérisation des épais- seurs et des indices de diélectriques ou de métaux, de couches antireflet, de surfaces polarisantes...

- La physique du solide : mesure de la permittivité, la structure de bande d'un composé de semi conducteurs...

- La microélectronique : caractérisation du taux d'implantation, de la rugosité de surface...

- La chimie et la biologie : caractérisation de liquides, d'interfaces liquide-solide, liquide-liquide [5].

- La métallurgie : analyse des alliages, mesure de concentration...

\section{Applications et}

\section{Photo de l'ellipsomètre}

Notre travail a été consacré au paramètre clé qui caractérise le silicium poreux SiP qui n'est autre que la porosité, et son influence sur les différents paramètres de ce matériau.

Pour notre échantillon SiP10, conditions de formation : $\mathrm{J}=10 \mathrm{~mA} / \mathrm{cm}^{2}, \mathrm{t}=5 \mathrm{~min}$. La porosité initiale de notre échantillon est égale à $65 \%$ [2], le programme de simulation nous donne : les parties réelles, $n$ et imaginaires $k$ du coefficient de réfraction, soit : $n=1.626070$ et $k=0.003037$.

En se basant sur les données fournies par ce programme de simulation on a voulu voir la relation entre la porosité initiale et la partie réelle et imaginaire de l'indice de réfraction $\mathrm{n}$ du silicium poreux (voir figs. $8 a$ et $b$ ).

\section{interprétations $[9,10]$}

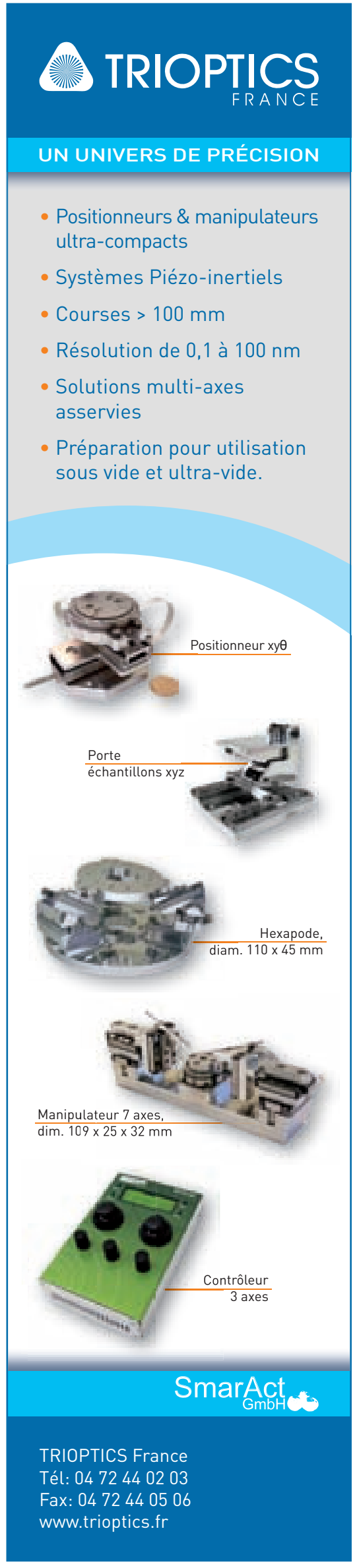

janvier/février 2011 • 51 • Thotoniques 
On constate d'après les résultats obtenus que la partie réelle du coefficient de réfraction diminue avec la porosité.

Le modèle de Bruggeman à 2 composantes est connu à être en bon accord avec les données expérimentales pour les couches de SiP de substrat $p$ Si de faible résistivité [2].

Afin de déterminer la porosité, l'indice de réfraction $n$ et la composante des films de SiP, nous utilisons la caractérisation par ellipsométrie, qui permet d'analyser les propriétés optiques et structurales des couches minces avec haute précision. Le calcul de l'indice $n$, de la porosité du SiP en fonction du degré d'oxydation du substrat Si en utilisant la modélisation de Bruggeman (modèle du milieu effectif). Les figures 9 et 10 représentent le degré $d^{\prime}$ oxydation de carcasse de silicium (s) et la porosité $(P)$ du silicium poreux SiP en fonction de l'indice de réfraction effective $n$.

La figure 9 montre que l'oxydation de SiP diminue avec l'augmentation de l'indice de réfraction effectif $n$ de SiP. Ceci s'explique par le fait que les pores sont occupés par la substance $\mathrm{SiO}_{2}$, avec $n_{\text {sio } 2}>1$. Donc il y a diminution de la carcasse du silicium puisque une partie a été oxydée. On constate que pour les films de faible porosité initiale, leur indice de réfraction effectif $n$ change après l'oxydation dans un large intervalle, tandis que pour les films de grandes porosités, I'indice de

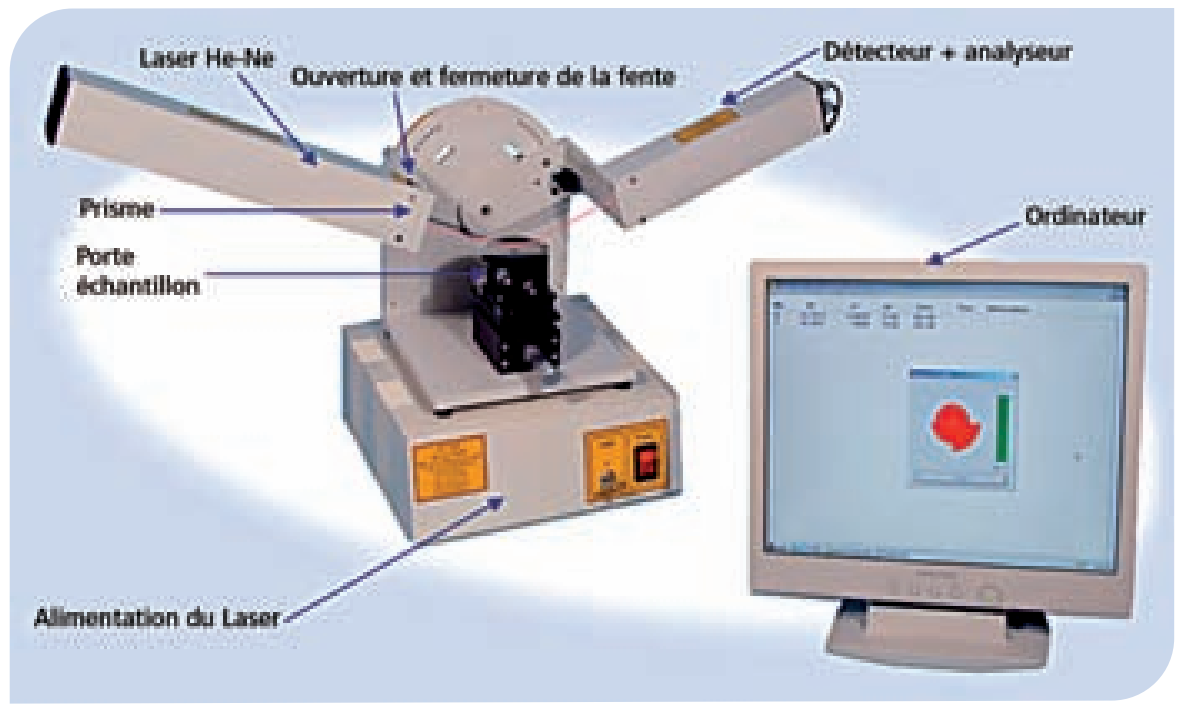

Figure 7. L'ellipsomètre ELX-1/02C.

réfraction $n$ est moins sensible au degré d'oxydation.

La figure 10 montre que les valeurs de l'indice de réfraction $n$ et de la porosité se trouvent dans l'air limité par deux courbes correspondantes au SiP à deux composantes:

- La courbe du haut correspond à un milieu contenant le silicium et le vide $(\mathrm{Si}+\mathrm{V})$.

- La courbe du bas correspond au système $\left(\mathrm{SiO}_{2}+\mathrm{V}\right)$.

On constate sur l'ensemble des courbes que pour différents indices de réfraction $n$ du silicium poreux, on peut tirer la porosité $p$ par la projection entre les deux courbes $(\mathrm{Si}+\mathrm{V})$ et $\left(\mathrm{SiO}_{2}+\mathrm{V}\right)$.
Les films du SiP avec une porosité initiale supérieure à $56 \%\left(P_{\text {in }}>56 \%\right)$ restent poreux même après une oxydation complète. Cependant, ceux d'une porosité initiale faible peuvent perdre la structure des pores, et se transformer en un autre système de deux composantes $\left(\mathrm{Si}+\mathrm{SiO}_{2}\right)$ (voir fig. 9).

\section{Conclusion}

L'ellipsométrie a fait preuve de sa puissance et de sa polyvalence dans plusieurs domaines aussi variés que la physique, la chimie, l'électrochimie, l'électronique, la verrerie, la mécanique de précision, les télécommunications, la biologie, les écrans plats.
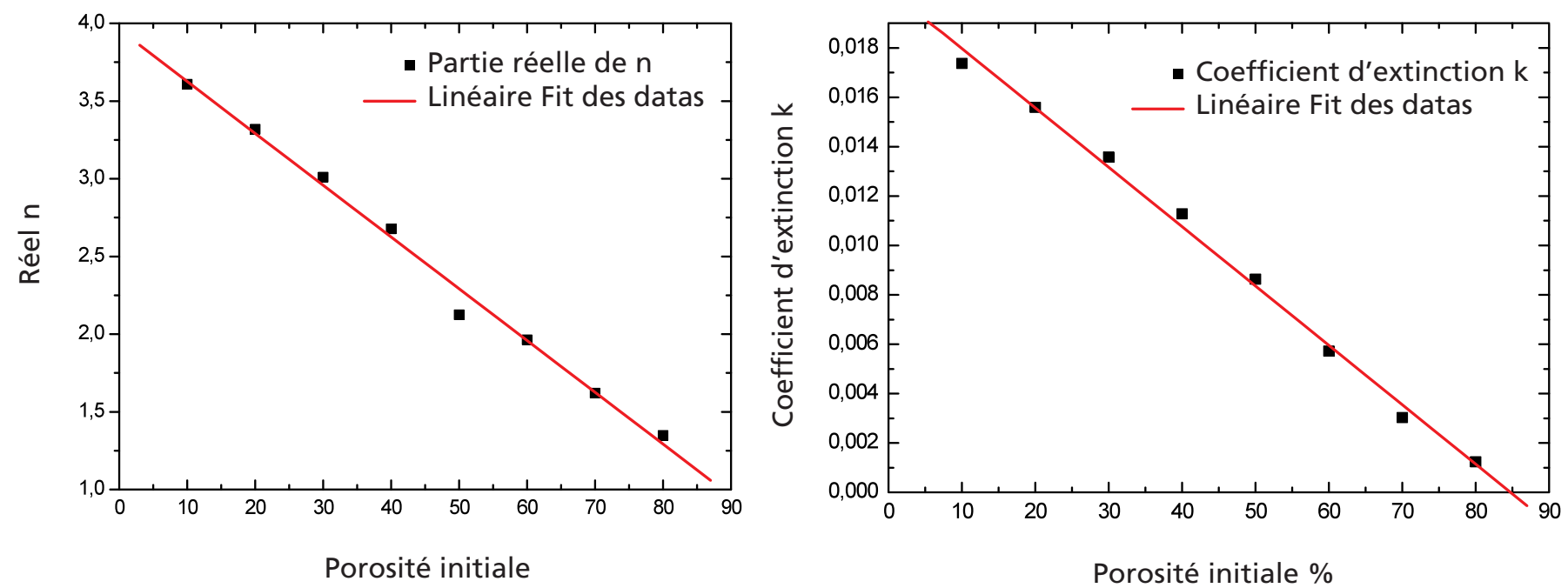

Figure 8a. Partie réelle du coefficient de réfraction en fonction de la porosité.

Figure 8b. Coefficient d'extinction en fonction de la porosité. 


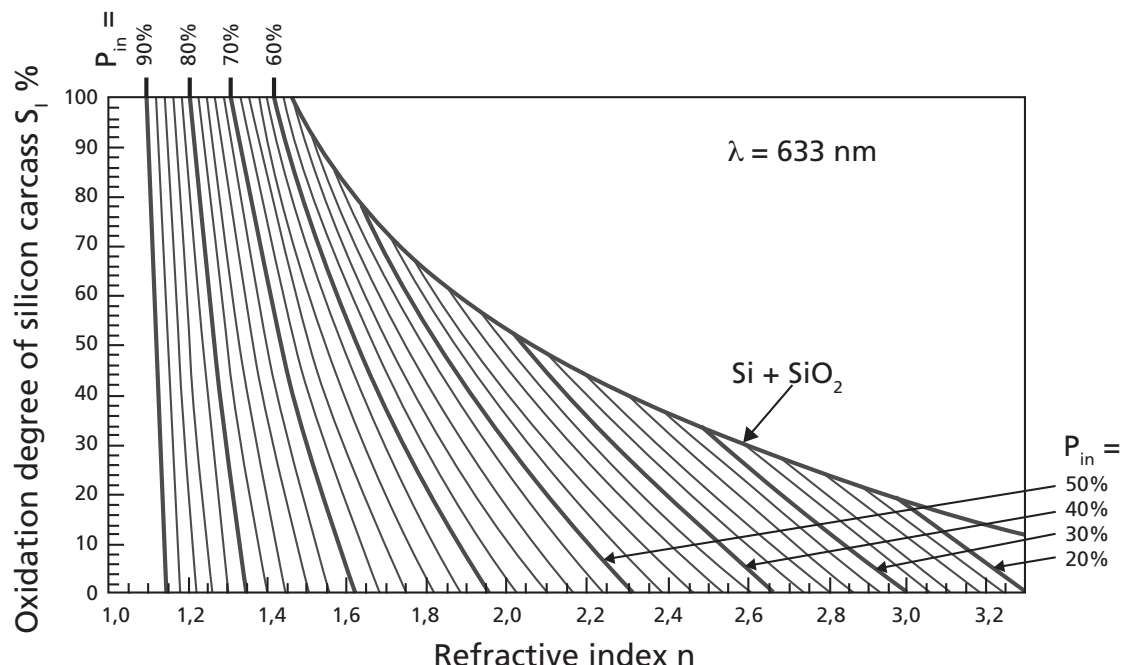

Figure 9. La relation entre le degré d'oxydation de carcasse de silicium (s) en fonction de l'indice de réfraction effective de silicium poreux $(n)$ pour des films de différentes porosités initiales $P_{i n}$.

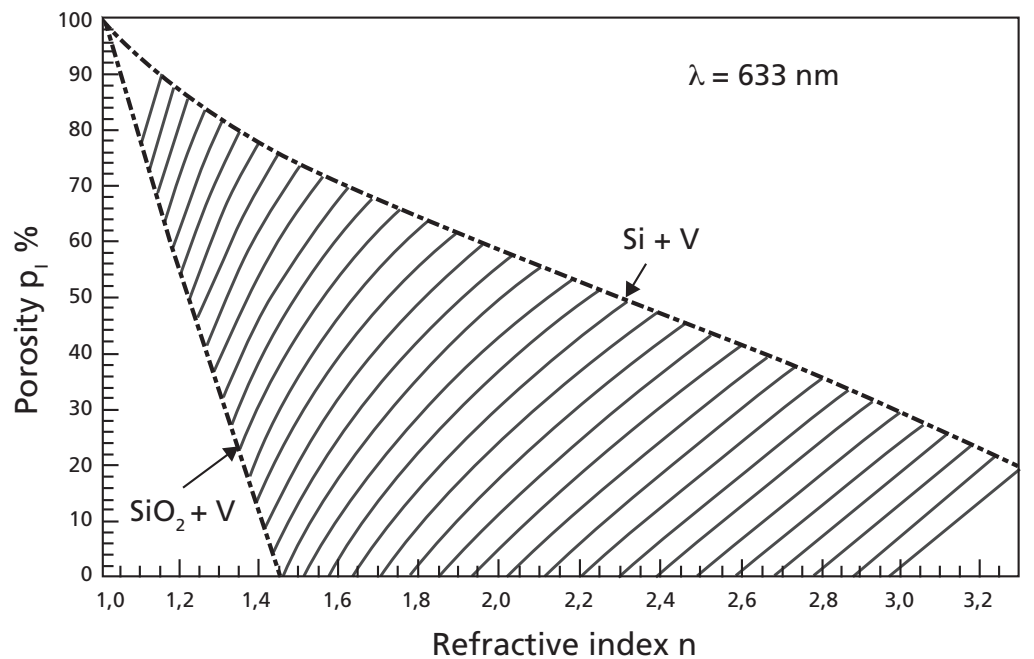

Figure 10. Relation entre l'indice de réfraction et la porosité des films oxydés de silicium poreux.

Elle est sollicitée à tous les stades de la haute technologie et de la R\&D. Le développement des nouveaux matériaux, des dispositifs d'affichage, les nanotechnologies, les MEMS (micro-electromechanical systems) et la réduction des dimensions des transistors, sont des facteurs qui feront de l'ellipsométrie une technique de métrologie de choix dans l'avenir.

Voir aussi la revue "Les technologies de laboratoires », N¹3, janvier-février 2009

\section{Références}

[1] R.M.A. Azzam and N.M. Bashara, in "Ellipsometry and polarized light", North Holland, 1986.

[2] P. Drude. Annalen der Physik 32.584 (1987):36.532 (1889):39.481 (1890).

[3] F. Bernoux, J.-P. Piel, J.-H. Lecat et J-L. Stehlé, in «Ellipsométrie» R 6490. Techniques de l'ingénieur traité mesures et contrôle.
[4] B. Lecourt «Ellipsométrie spectroscopique conventionnelle et généralisée de films moléculaires ultra minces ». Thèse de doctorat de l'Université Bordeaux. 1998.

[5] M.-H. Debroux, Ph. Ged,A.Vareille, in « Ellipsométrie spectroscopique ». L'Écho des recherches. 1983.

[6] B. Castellon, in «Ellipsométrie spectroscopique, principe et application». Photonique la revue des solutions optiques. 2002.

[7] B. Drévillon, "Spectroscopic ellipsometry in the infrared range", Thin Solid Films 313-314 (1998), pp. 625-630.

[8] N. Bertrand, P. Bulkin, B. Drévillon, S. Lucas, S. Benayoun, "In situ infrared ellipsometry study of plas ma processing of metallic surfaces", Surface and Coatings Technology, vol. 94-95 (1997), pp. 362-367.

[9] N. Ghellai, « Caractérisations des couches nanoporeuses de silicium poreux et leurs applications. Cas de la détection de gaz ». Thèse de doctorat d'état de I'Université de Tlemcen. 2006.

[10] E.V.Astrova, V.A. Tolmochev, Materiels Science and Engineering B 69-70, pp. 142-148 (2000).

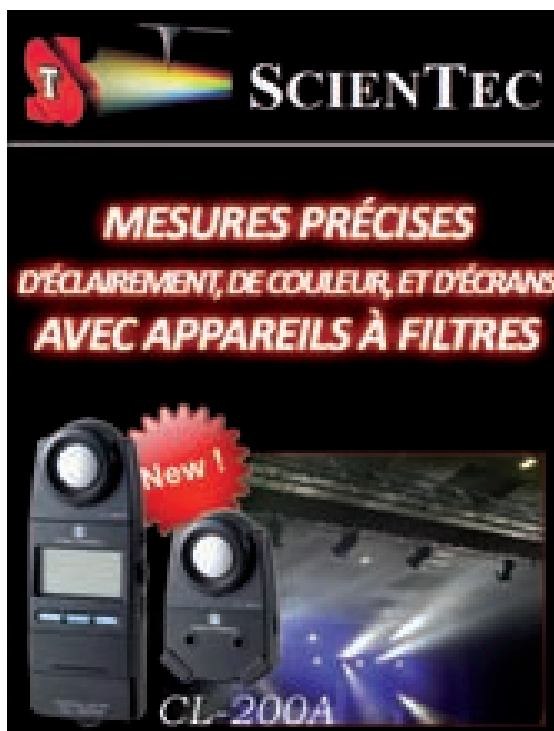

Luxmètre chromamètre

Echarage intericur et extricur des

batiments, mesure de sources

lumineuses, mesure de $L E D$, eabines

de lumiere,

- Mesures en lux et en couleur

- Temperature de couleur

- Longueur donde, purrté dexcitation

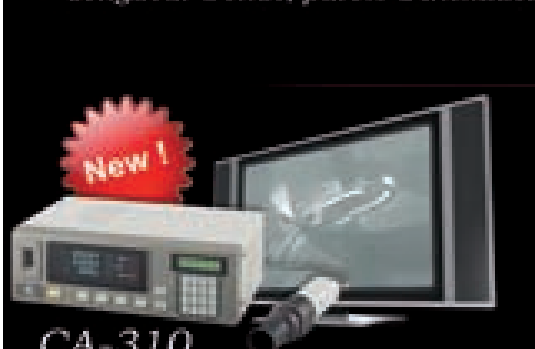

CA-310

Analyseur d'écrans

LCD, TFT, CRT, PLASMA, FED, OLED, DLP.

- Mesures en luminance $(\mathrm{cd} / \mathrm{m} / \mathrm{m}$

coulear, temperature de couleur

- Controle qualité en chromaticité

- Ajustage balance de blanc

\section{ScienTec,} c'est aussi..

Spectroradiomètres

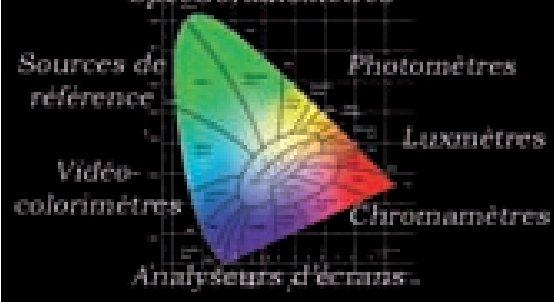

Tel : +33 (0) 1,64..63,27,00

infoes seicatec,fr / WwW-urientec-ff 